\title{
Characterization of PCL and Chitosan Nanoparticles as Carriers of Enoxaparin and Its Antithrombotic Effect in Animal Models of Venous Thrombosis
}

\author{
Lucas Bessa Prado, ${ }^{1}$ Stephany Cares Huber, ${ }^{1}$ Aline Barnabé, ${ }^{1}$ \\ Fernanda Dutra Santiago Bassora, ${ }^{1}$ Devanira Souza Paixão, ${ }^{1}$ \\ Nelson Duran, ${ }^{2}$ and Joyce Maria Annichino-Bizzacchi ${ }^{1}$ \\ ${ }^{1}$ Department of Medical Pathophysiology and Hematology and Hemotherapy Center, University of Campinas/Hemocentro-Unicamp, \\ Instituto Nacional de Ciência e Tecnologia do Sangue, Campinas, SP, Brazil \\ ${ }^{2}$ Department of Physical Chemistry, University of Campinas, Campinas, SP, Brazil
}

Correspondence should be addressed to Stephany Cares Huber; huber@unicamp.br

Received 11 October 2016; Revised 1 February 2017; Accepted 2 February 2017; Published 23 February 2017

Academic Editor: Shih-Feng Chou

Copyright ( 2017 Lucas Bessa Prado et al. This is an open access article distributed under the Creative Commons Attribution License, which permits unrestricted use, distribution, and reproduction in any medium, provided the original work is properly cited.

\begin{abstract}
This study was based on the preparation, characterization, and animal in vivo experiments performed to evaluate nanoparticles of poly( $\varepsilon$-caprolactone) (PCL) and chitosan as carriers of enoxaparin. The nanoparticles were characterized and presented satisfactory results in terms of size, polydispersity, and encapsulation efficiency. Anticoagulant activity of the nanoparticles was maintained for 14 hours when the administration was subcutaneous; however no activity was observed after oral administration. There was a significant reduction in thrombus size, in vivo, for both free and encapsulated enoxaparin in comparison with the control group after subcutaneous administration. Oral administration results however were indifferent. In conclusion, the double emulsion method w/o/w was efficient for enoxaparin encapsulation, producing spherical nanoparticles with high encapsulation efficiency. For in vivo studies, the encapsulated enoxaparin showed a sustained anticoagulant activity for a higher period of time compared to free enoxaparin, with an antithrombotic effect when administered subcutaneously.
\end{abstract}

\section{Introduction}

Deep vein thrombosis (DVT) is one of the most important public health problems, with an annual incidence estimated as 67 per 100.000 individuals in the population [1,2]. Heparin is a drug with antithrombotic action, widely used since 1930 [3]. Low molecular weight heparin (LMWH) exhibits superior pharmacodynamic and pharmacokinetic properties compared to heparin and shows low affinity for plasma and cellular proteins. Therefore, LMWH exhibited a more predictable and reproducible anticoagulant response than heparin and did not require monitoring in most clinical situations [4].

The new direct oral anticoagulants available present limitations of antidotes for anti-Xa drugs and lack laboratory tests which would be used in special situations such as bleeding or emergency procedures [5]. Adherence to treatment is indeed a challenge in clinical practice for medications with continuous daily use. Therefore, the possibility of drugs with a prolonged half-life which do not increase bleeding represent a good opportunity for a better management of those patients.

A sustained release drug is intended to maintain the effect of the active component in the target tissue, by solubilizing this active component, to improve the physical and chemical stability of therapeutic agents, minimize side effects, and reduce toxicity [6-8]. In a sustained release system, the active ingredient encapsulated in a matrix must have the following characteristics: enabling modeling of the desired shape and release of the active substance by appropriate mechanisms, adequate stability, and low toxicity including any degradation product $[8,9]$. Furthermore, reduced side effects, increased bioavailability, and decreased number of required doses of the therapeutic agent are important issues $[9,10]$. PCL is 
semicrystalline polyester, biodegradable, and biocompatible. This, along with the mechanical properties and relatively low cost of PCL, renders this polymer very attractive for biomedical devices [11].

In the pharmaceutical area, chitosan has been widely used in drug delivery systems [12]. In addition to being a biocompatible polymer, biodegradable, and nontoxic, chitosan has interesting biological properties such as antimicrobial activity, potent topical analgesic action, acceleration of the healing process, and bioadhesion to mucous membranes and skin [13].

The preparation of heparin encapsulated in nanoparticles by the double emulsion method water in oil in water $(\mathrm{w} / \mathrm{o} / \mathrm{w})$ using biodegradable polymers such as PCL and PLGA alone or in combination with other biodegradable polymers (Eudragit RS and RL) has been successfully used. These studies showed reproducible results regarding diameter, encapsulation efficiency, and anti-Xa activity in vitro and in animal models [14-17].

The use of animal models to investigate DVT is advantageous enabling the evaluation of new pharmaceutical approaches for prophylaxis and treatment and of how these approaches can interfere in initiation, propagation, and resolution of venous thrombus.

In this study we produced and characterized nanoparticles of PCL and chitosan as carriers of LMWH, enoxaparin. We also investigated the antithrombotic effect through oral and subcutaneous administration in animal models of venous thrombosis.

\section{Methods}

2.1. Preparation of Chitosan-PCL Nanoparticles with Enoxaparin. Nanoparticles were prepared with a biodegradable polymer PCL (Aldrich) and coated with the natural polymer chitosan (105 g/mol/ 81\% acetylation, Polymar). The preparation of nanoparticles with and without enoxaparin was performed by the modified double emulsion method. An aqueous solution $(200 \mu \mathrm{l})$ of enoxaparin (Clexane $100 \mathrm{mg} / \mathrm{mL}$ ), $1 \mathrm{~mL}$ of deionized water, and $0.5 \%$ of polyvinyl alcohol (PVA) was first emulsified with an organic phase containing $10 \mathrm{~mL}$ of ethyl acetate and $60 \mathrm{mg}$ of PCL under stirring for 1 minute at $6000 \mathrm{rpm}$ to form a water/oil emulsion. This emulsion was then transferred to a vessel containing $50.1 \mathrm{ml}$ of deionized water, $1 \%$ PVA, $0.9 \% \mathrm{NaCl}$, and $9.9 \mathrm{~mL}$ of chitosan solution $(2 \mathrm{mg} / \mathrm{mL})$ and homogenized under mechanical stirring at $10000 \mathrm{rpm}$ for 1.5 minutes, forming a double emulsion (water/oil/water). After this period the double emulsion was transferred to a round bottom flask and the solvent evaporated at a temperature of $28^{\circ} \mathrm{C}$ in a route evaporator to a final volume of $10 \mathrm{~mL}$.

2.2. Particle Size and Zeta Potential. The average particle size, polydispersity index (PDI), and zeta potential were measured through photon correlation spectroscopy (PCS) by Zeta Sizer Malvern (Malvern Instruments Corp). Measurements were performed in $\mathrm{KCl}$ solution $(1 \mathrm{mM})$. The preparation stability was measured weekly; for this, the dispersions was maintained at $4^{\circ} \mathrm{C}$ during 50 days.
2.3. Scanning Electron Microscopy (SEM). The particles were characterized by Scanning Electron Microscopy (SEM, Jeol JSM-6360LV) at a voltage of $20 \mathrm{kV}$ after prior coating with gold/palladium under vacuum by sputtering using a BALTEC apparatus. Secondary electron images were obtained.

2.4. Encapsulation Efficiency. The encapsulation efficiency (EE) of enoxaparin encapsulated in PCL-chitosan nanoparticles was determined by the modified Azure II colorimetric method [18]. Particle dispersion was filtered in a Microcon centrifugal filter device containing ultrafiltration membranes (MWCO 100.000, Millipore). The filtrate was mixed with Azure II solution $(0.05 \mathrm{mg} / \mathrm{mL})(1: 10)$ at room temperature and the sample was measured at $\lambda=500 \mathrm{~nm}$ using UV-Vis spectrophotometer (Agilent 8453). The filtrate was assayed to determine the concentration of the nonencapsulated drug. Encapsulation efficiency (EE\%) was calculated using the following equations [16]:

$\%$ non-encapsulated drug

$$
=\left(\frac{[\text { enoxaparin }] \text { in filtered solution }}{[\text { enoxaparin }] \text { initial }}\right) \times 100 \text {, }
$$

$(\mathrm{EE} \%)=100-\%$ non-encapsulated enoxaparin.

2.5. Anticoagulant Action Measured by Anti-Xa Activity in Normal Rats. Male Wistar rats, from CEMIB-UNICAMP, were used respecting the Brazilian Law of Animal Experiments. The experimental protocol was approved by the University Committee on Animal Research.

The experiments were performed with 32 male Wistar rats at 5-7 weeks of age, divided into four groups. The aqueous enoxaparin suspension encapsulated in PCL-chitosan nanoparticles $(5000 \mathrm{IU} / \mathrm{kg})$, empty nanoparticles $(5000 \mathrm{IU} / \mathrm{Kg})$, free enoxaparin (500 IU/Kg), and saline solution were administered orally or by subcutaneous injection. The anti-Xa activity (Top-IEL 500) was determined in plasma samples at $1,2,3,6,8,10,12$, and 14 hours after administration in each group. Blood samples were withdrawn from the tail vein into a vial containing sodium citrate, which was gently mixed before centrifugation for 15 minutes at $600 \mathrm{~g}$ to obtain plasma.

Anti-Xa activity was determined by a chromogenic substrate assay (Berichrom Heparin, Siemens), with detection limit of $0.05 \mathrm{IU} / \mathrm{mL}$. In this evaluation, enoxaparin was analyzed as a complex with antithrombin present in the sample. The concentration of this complex is dependent on the availability of the endogenous antithrombin in the animal. Factor $\mathrm{Xa}$ is neutralized by the antithrombin-enoxaparin complex. The residual Xa factor is quantified using a synthetic chromogenic substrate.

2.6. Antithrombotic Activity in a Venous Thrombosis Animal Model. The experiments were performed with 48 male Wistar rats of 5-7 weeks of age, divided into four groups of six animals. The animals were anesthetized by intraperitoneal injection of a solution of ketamine $(75 \mathrm{mg} / \mathrm{Kg}$ ) (Ketalar, ParkeDavis, Brazil) and xylazine $(10 \mathrm{mg} / \mathrm{Kg}$ ) (União Química Farmacêutica Nacional S/A, Brazil). 
TABLE 1: Values of diameter, polydispersity index, and zeta potential of three dispersions of enoxaparin nanoparticles.

\begin{tabular}{lccc}
\hline Particles dispersion & Diameter $(\mathrm{nm})$ & Polydispersity index & Zeta potential $(\mathrm{mV})$ \\
\hline 1 & 512.5 & 0.458 & +30.7 \\
2 & 526.8 & 0.401 & +32.3 \\
3 & 499.2 & 0.369 & +29.8 \\
\hline Mean & 512.8 & 0.409 & +30.9 \\
Standard deviation & 13.8 & 0.04 & 1.3 \\
\hline
\end{tabular}

Enoxaparin encapsulated in PCL-chitosan nanoparticles (5000 IU/kg), empty nanoparticles, free enoxaparin (500 IU/ $\mathrm{Kg}$ ), and saline solution were administered subcutaneously or orally. One hour (subcutaneous) or six hours (oral) after administration, the abdomen of the animals was surgically opened and thrombosis was induced according to the modified method of Reyers et al. (1980) and Carvalho et al. (1992) and modified method of Herbert et al. (1992) [19-21]. Briefly, the cava vein was isolated and its branches were ligated up to $1.5 \mathrm{~cm}$ distal to the left renal vein confluence. Ligature of the cava vein was carried out using 4-0 silk thread at a point immediately caudal to the left renal vein. After ligation, thromboplastin solution (Thromborel ${ }^{\circledR}$ S; Siemens; $\leq 60 \mathrm{mg}$ / $\mathrm{mL}$ ) was injected at a concentration of $5 \mathrm{mg} / \mathrm{kg}$ body weight of the animal in the attached segment. Twenty minutes after induction, the abdomen was opened to verify the presence of thrombus. When present, the thrombus was removed and weighed immediately and then after drying for $24 \mathrm{~h}$ at $37^{\circ} \mathrm{C}$.

2.7. Statistical Analysis. The results of encapsulation efficiency, average diameter, and zeta potential were analyzed and reported as mean \pm standard deviation.

Anti-Xa activity and comparison between 4 independent groups were analyzed by the nonparametric Kruskal-Wallis test; a value of $P<0.05$ was considered statistically significant.

\section{Results}

3.1. Preparation of Chitosan-PCL Nanoparticles with Enoxaparin and Stability. Table 1 shows the characterization of PCL-chitosan nanoparticles with enoxaparin. The double emulsion and solvent evaporation method showed being reproducible.

Furthermore, there were no significant changes in the size and zeta potential of PCL-chitosan nanoparticles with and without enoxaparin, evidencing the stability of these particles for a period of 50 days at $4^{\circ} \mathrm{C}$, as shown in Figures 1(a) and 1(b). This result demonstrates that both particles are good carrier systems. The PCL-chitosan nanoparticles produced by $w / o / w$ double emulsion showed an entrapment efficiency of $99.04 \pm 0.001 \%$, therefore demonstrating being a good method for hydrophilic drug encapsulation.

The SEM images of the PCL nanoparticles showed spherical and homogeneous particles (Figure 2). The morphology did not change after enoxaparin encapsulation, as shown in Figure 2(b).
3.2. Anticoagulant Activity Measured by Anti-Xa Activity in Normal Rats. Figure 3 shows the results of anti-Xa activity in rat plasma samples collected in determined periods after subcutaneous injection of saline solution, free enoxaparin (500 UI/Kg), and nanoparticles with and without enoxaparin (5000 UI/Kg). After administration of free enoxaparin there was a peak of anti-Xa activity after 1 hour $(0.37 \mathrm{IU} / \mathrm{ml})$, which disappeared within 6 hours. When the PCL-chitosan nanoparticles with enoxaparin were administered, there was a peak of anti-Xa activity after 3 hours $(1.8 \mathrm{IU} / \mathrm{ml})$, which was much higher than that obtained with free enoxaparin and remained constant for 10 hours. This result was significantly higher when compared to free enoxaparin $(P=0.0043)$. After 14 hours a remaining anti-Xa activity of $0.6 \mathrm{IU} / \mathrm{mL}$ was observed. Empty nanoparticles (without enoxaparin) exhibit no anti-Xa activity, identical to that observed with saline solution.

For all groups evaluated, oral administration showed no anti-Xa activity in rats plasma, indicating no antithrombotic effect of these formulations.

3.3. Antithrombotic Effect in a Venous Thrombosis Animal Model. Figure 4 shows the analysis of the antithrombotic effect, as measured by thrombus weight, in animals treated with saline solution, free enoxaparin, and nanoparticles with enoxaparin $(50 \mathrm{mg} / \mathrm{Kg})$ administered subcutaneously. There was a significant lower thrombus weight in the treated groups in comparison to control $(P=0.0005)$ (Figure 4).

Figure 5 shows the analysis of the antithrombotic effect, as assessed by thrombus weight after animals were treated orally with saline solution, empty nanoparticles, and nanoparticles with enoxaparin. The results showed a similar thrombi weight and no significant difference between the groups $(P=$ 0.0757).

\section{Discussion}

The double emulsion method and solvent evaporation used in this study were able to produce relatively homogeneous and spherical nanoparticles. These properties are important for nanoparticle quality. A major difficulty encountered during enoxaparin encapsulation is the hydrophilic nature of this anticoagulant, which leads to greater diffusion of the drug to the external phase before the precipitation of the polymer, thus reducing encapsulation efficiency [22]. The comparison between the size of our nanoparticles and those described in another study showed similar results [23]. The nanoparticles 

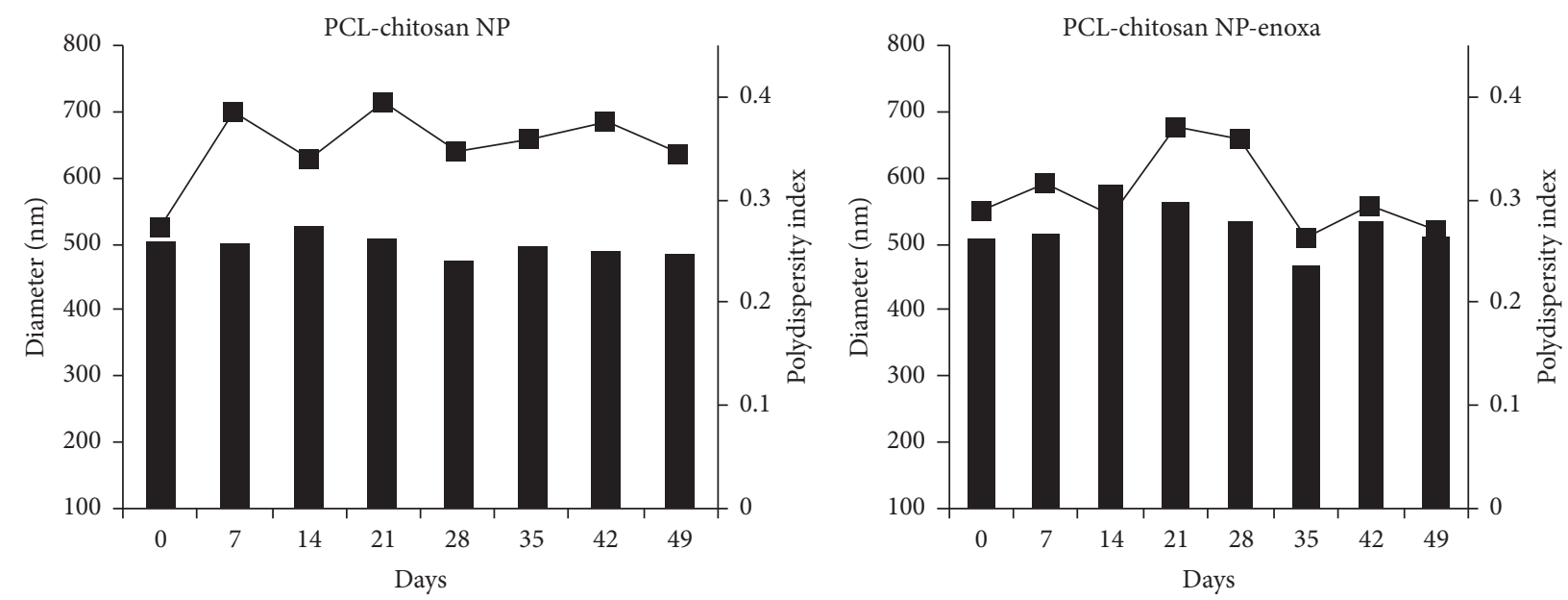

Diameter
- Polydispersity index
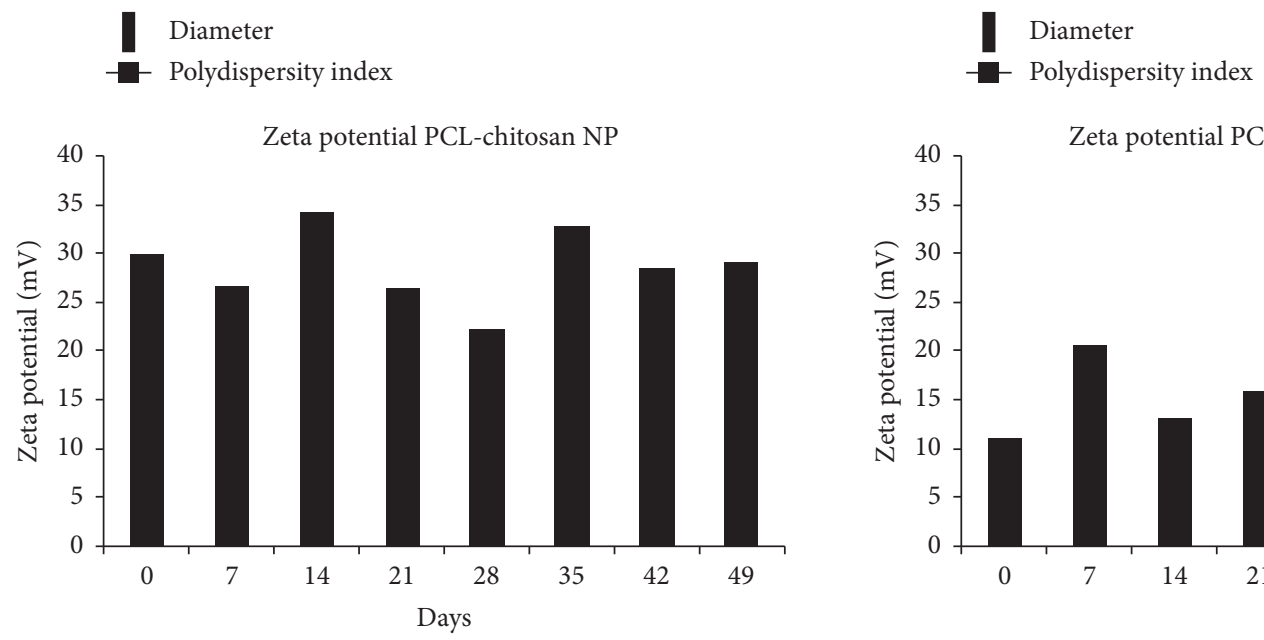

(a)

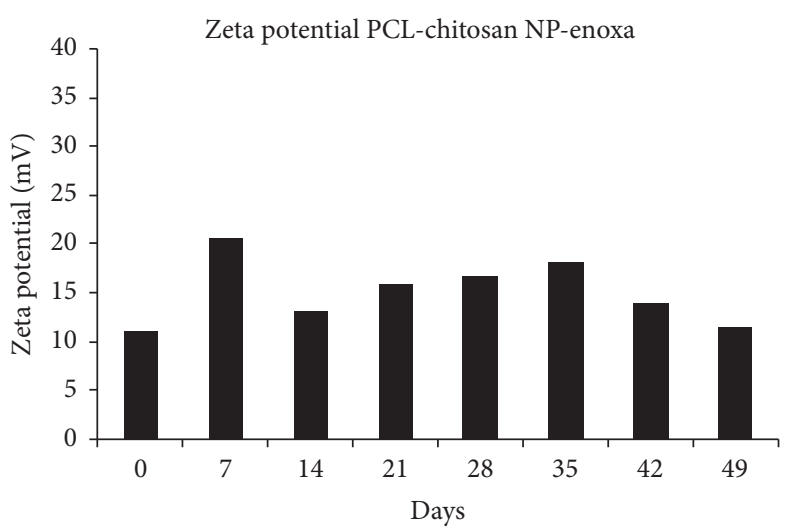

(b)

FIGURE 1: Diameter, polydispersity index, and zeta potential of the nanoparticles during 50 days: (a) empty nanoparticles; (b) nanoparticles containing enoxaparin.

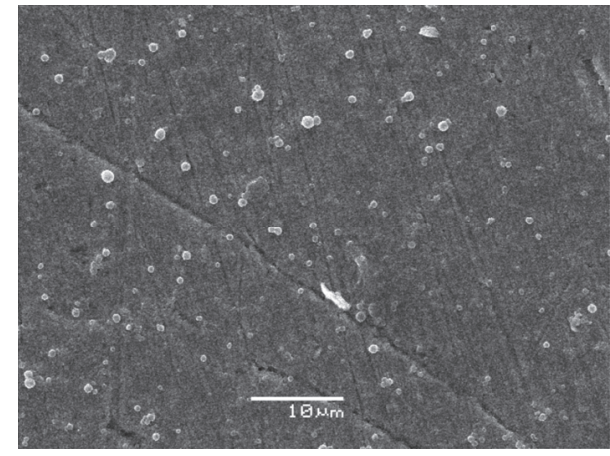

(a)

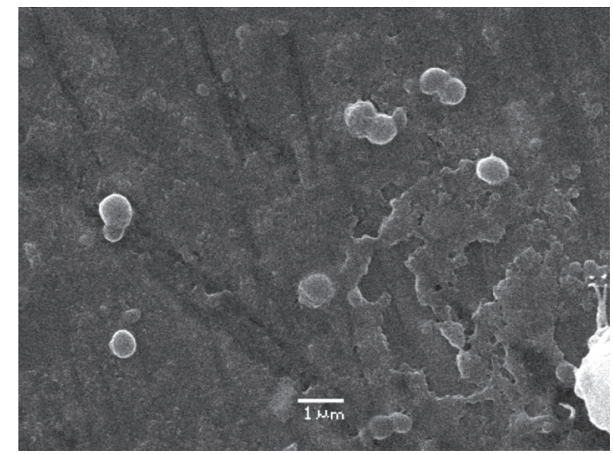

(b)

FIGURE 2: SEM microscopy of nanoparticles: (a) empty nanoparticles; (b) nanoparticles containing enoxaparin.

were stable for over 50 days regarding size and polydispersity index. Regarding encapsulation efficiency, results of $99.04 \%$ on average reinforce the idea that the natural polymer chitosan used for coating the nanoparticles is very appropriate for encapsulation of hydrophilic molecules, especially when this dispersion of particles comes into contact with the stomach mucosa, which has acid $\mathrm{pH}$.

Our in vivo results evidenced that anti-Xa activity was higher with the use of nanoparticles containing enoxaparin in comparison to free enoxaparin. A possibility is that the dose 


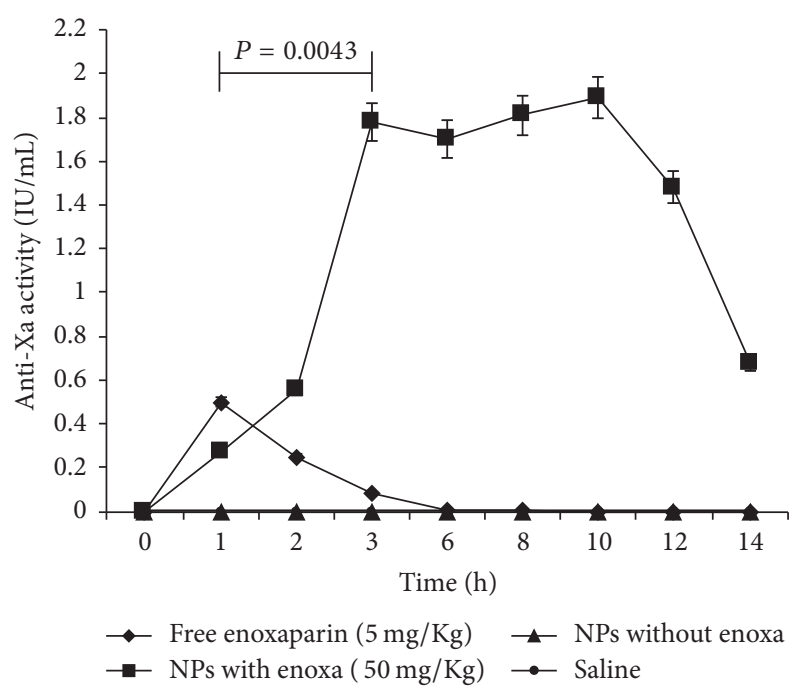

FIgURE 3: Anti-Xa activity in relation to time after subcutaneous application of free enoxaparin, encapsulated enoxaparin, empty nanoparticles, and saline in the groups. $N=16$ animals, 4 per group. $P$ value calculated through Mann-Whitney test. NPs: nanoparticles.

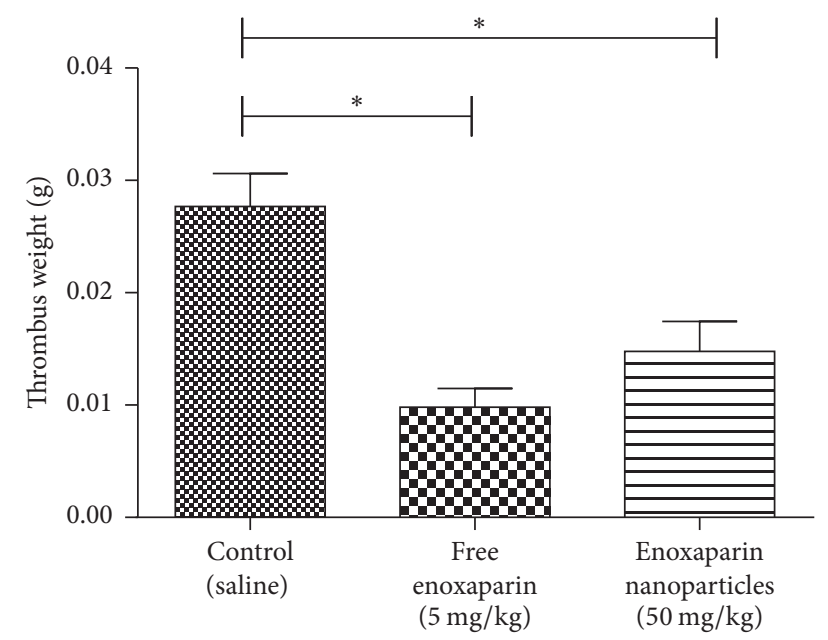

FIGURE 4: Antithrombotic effect shown through the comparison of thrombus weight of animals after surgery and subcutaneous treatment ( $n=6$ animals per group). ${ }^{*} P=0.0005$. $P$ value was calculated using ANOVA.

of enoxaparin used in the encapsulated nanoparticles was 10 times higher than the dose of free enoxaparin. We elected this high dose of enoxaparin as encapsulation could compromise the stability, or a sustained and prolonged release could require a higher concentration of the drug. We have previously demonstrated a slow release of encapsulated PCLenoxaparin in vitro during the first 24 hours [17].

The controlled release characteristic of nanomaterials enabling drug liberation for a long period of time and preserving the pharmacological effect without reaching the toxicity range is interesting. This result of prolonged anti-Xa

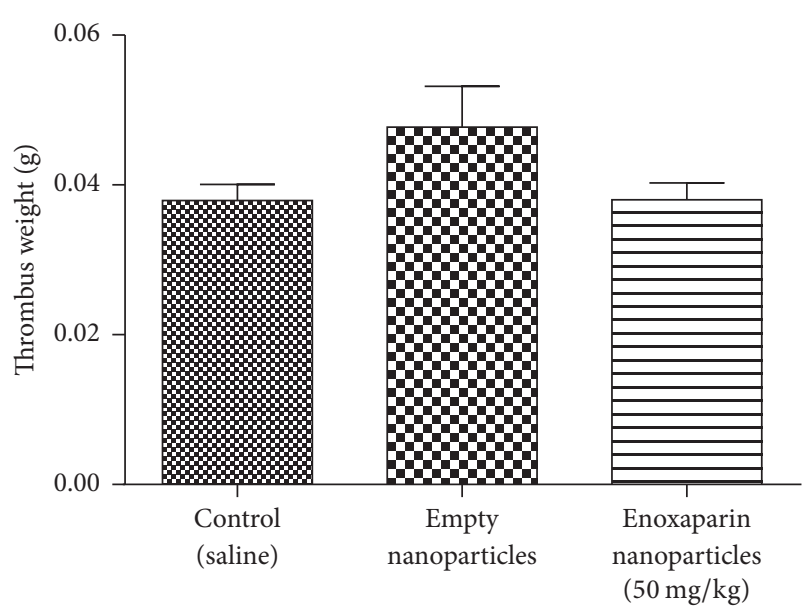

FIGURE 5: Comparison of thrombus weight in animals after surgery and oral treatment. $N=6$ animals per group. $P$ value calculated through ANOVA.

activity with the use of enoxaparin encapsulated in nanoparticles should be valuable, as a drug that has a preserved survival rate in safe doses is desirable in clinical practice.

The oral administration of enoxaparin, free or encapsulated into nanoparticles, demonstrated no anticoagulant effect, with no anti-Xa activity up to 14 hours, suggesting that enoxaparin was not absorbed along the gastrointestinal tract by premature degradation or failure in absorption [24].

Thus, our results demonstrate that encapsulation of enoxaparin in PCL nanoparticles with chitosan was not able to avoid this degradation process or provide gastrointestinal absorption. Sun et al. (2010) recently reported that complexes of polyelectrolytes with chitosan and enoxaparin with similar diameters provided an anticoagulant activity in rats, when administered orally [23]. However, aPTT was the method used to measure anticoagulant activity of nanoparticles and showed an increase of only 2 seconds when compared to the saline control. The results did not include the concentration of enoxaparin nanoparticles either. Moreover, a study using PCL, PVA, and Eudragit nanoparticles with tinzaparin (200 to $600 \mathrm{IU} / \mathrm{Kg}$ ) with a diameter of approximately $400 \mathrm{~nm}$ showed oral absorption and anticoagulant activity in rabbits, demonstrated by an increase in anti-Xa activity in plasma [15]. Jiao et al. (2002) published a study using heparin and PCL and PLGA and Eudragit RS and/or RL, obtaining minor particles for oral delivery in rabbits. These nanoparticles were shown to be functional verified through anti-Xa activity and aPTT [16]. These results suggested that Eudragit or modified chitosan could probably contribute decisively to oral absorption. Pazzini et al. (2015) published a study using PCL and enoxaparin for subcutaneous route. Beyond differences in the particle properties, such as negative potential and no use of chitosan, some differences in the biological activity were verified [17]. The PCL coated chitosan was more efficient for reaching the maximum of activity at $3 \mathrm{~h}(1.8 \mathrm{anti}-\mathrm{Xa} \mathrm{UI} / \mathrm{mL})$ and without chitosan the maximum value was at $6 \mathrm{~h}(0.6 \mathrm{anti}-$ $\mathrm{Xa} \mathrm{UI} / \mathrm{mL}$ ). The release in the first case lasted approximately $14 \mathrm{~h}$ and in the second one zero at that time. 
Induction of DVT using models of stasis and hypercoagulability enables the development of homogeneous and reproducible thrombi, accelerating the process, which facilitates the analysis of the effect of antithrombotic drugs. In our study the reduction of thrombus size after the use of subcutaneous nanoparticles with enoxaparin confirmed the in vivo antithrombotic effect. This result is important for excluding the hypothesis of no effect of enoxaparin nanoparticles administered orally due to the encapsulation.

Our results also demonstrated that oral administration of free or encapsulated enoxaparin presented no antithrombotic effect. These results confirm the laboratorial effects measured by anti-Xa activity observed in these animal models. Therefore, these results reinforce the hypothesis that enoxaparin was either prematurely degraded or not absorbed.

Therefore, particles with nanometric diameter $(500 \mathrm{~nm})$ and positively charged were obtained by the double emulsion method and solvent evaporation. The method was reproducible and, in addition, the obtained particles were stable for 50 days. High encapsulation efficiency ( 99\%) was obtained for the hydrophilic drug enoxaparin indicating that the method is suitable for this molecule encapsulation.

The study showed that nanoparticles with enoxaparin exhibit anticoagulant and antithrombotic activity in DVT rat models when administered subcutaneously.

The addition of chitosan did not provide an antithrombotic or anticoagulant effect for oral administration of these nanoparticles in rats. This result may be due to nonabsorption of nanoparticles or early degradation of enoxaparin in the gastrointestinal tract. In the literature, chitosan was used as an effective hemostatic agent in animal models of splenic and liver injury. Studies suggest that chitosan may enhance hemostasis by cell-cell contact or by the formation of a lattice, which entraps cells to form an artificial clot [25]. In our study, chitosan was used to recover the nanoparticles, with a minimum surface of contact with the blood stream due to nanostructures. Indeed, an in vivo evaluation with empty nanoparticles was performed and similar anti-Xa activity was observed when compared to controls (saline), suggesting no effects on hemostasis, as shown in Figure 3.

\section{Competing Interests}

The authors declare that there is no conflict of interests regarding the publication of this paper.

\section{Acknowledgments}

The authors would like to acknowledge FAPESP and CNPq and Brazilian Network on Nanocosmetics (MCT/CNPq) for financial support. They would also like to thank Priscyla D Marcato for her substantial contribution to this study.

\section{References}

[1] M. D. Silverstein, J. A. Heit, D. N. Mohr, T. M. Petterson, W. M. O'Fallon, and L. J. Melton III, "Trends in the incidence of deep vein thrombosis and pulmonary embolism: a 25 -year population-based study," Archives of Internal Medicine, vol. 158, no. 6, pp. 585-593, 1998.

[2] R. H. White, "The epidemiology of venous thromboembolism," Circulation, vol. 107, no. 23, p. -I8, 2003.

[3] F. Longhi, D. Laks, and N. G. N. Kalil, “Trombocitopenia induzida por heparina," Revista Brasileira de Hematologia e Hemoterapia, vol. 23, no. 2, pp. 93-99, 2001.

[4] J. W. Park, O. C. Jeon, S. K. Kim et al., "Pharmacokinetic evaluation of an oral tablet form of low-molecularweight heparin and deoxycholic acid conjugate as a novel oral anticoagulant," Thrombosis and Haemostasis, vol. 105, no. 6, pp. 1060-1071, 2011.

[5] R. Paliwal, S. R. Paliwal, G. P. Agrawal, and S. P. Vyas, "Chitosan nanoconstructs for improved oral delivery of low molecular weight heparin: in vitro and in vivo evaluation," International Journal of Pharmaceutics, vol. 422, no. 1-2, pp. 179-184, 2012.

[6] P. D. Marcato and N. Durán, "New aspects of nanopharmaceutical delivery systems," Journal of Nanoscience and Nanotechnology, vol. 8, no. 5, pp. 2216-2229, 2008.

[7] B.-B. C. Youan, A. Hussain, and N. T. Nguyen, "Evaluation of sucrose esters as alternative surfactants in microencapsulation of proteins by the solvent evaporation method," AAPS PharmSci, vol. 5, no. 2, 2003.

[8] S. Miyazaki, A. Takahashi, W. Kubo, J. Bachynsky, and R. Löbenberg, "Poly n-butylcyanoacrylate (PNBCA) nanocapsules as a carrier for NSAIDs: in vitro release and in vivo skin penetration," Journal of Pharmacy and Pharmaceutical Sciences, vol. 6, no. 2, pp. 240-245, 2003.

[9] J. Panyam and V. Labhasetwar, "Biodegradable nanoparticles for drug and gene delivery to cells and tissue," Advanced Drug Delivery Reviews, vol. 55, no. 3, pp. 329-347, 2003.

[10] K. S. Soppimath, T. M. Aminabhavi, A. R. Kulkarni, and W. E. Rudzinski, "Biodegradable polymeric nanoparticles as drug delivery devices," Journal of Controlled Release, vol. 70, no. 1-2, pp. 1-20, 2001.

[11] E. Luong-Van, L. Grøndahl, K. N. Chua, K. W. Leong, V. Nurcombe, and S. M. Cool, "Controlled release of heparin from poly( $\varepsilon$-caprolactone) electrospun fibers," Biomaterials, vol. 27, no. 9, pp. 2042-2050, 2006.

[12] A. A. M. Lira, D. M. A. Nanclares, A. F. Neto, and J. M. Marchetti, "Drug-polymer interaction in the all-trans retinoic acid release from chitosan microparticles," Journal of Thermal Analysis and Calorimetry, vol. 87, no. 3, pp. 899-903, 2007.

[13] H. S. Silva, K. S. Santos, and E. I. Ferreira, "Quitosana: derivados hidrossolúveis, aplicações farmacêuticas e avanços," Química Nova, vol. 29, no. 4, pp. 776-785, 2006.

[14] V. Hoffart, N. Ubrich, C. Simonin et al., "Low molecular weight heparin-loaded polymeric nanoparticles: formulation, characterization, and release characteristics," Drug Development and Industrial Pharmacy, vol. 28, no. 9, pp. 1091-1099, 2002.

[15] V. Hoffart, A. Lamprecht, P. Maincent, T. Lecompte, C. Vigneron, and N. Ubrich, "Oral bioavailability of a low molecular weight heparin using a polymeric delivery system," Journal of Controlled Release, vol. 113, no. 1, pp. 38-42, 2006.

[16] Y. Jiao, N. Ubrich, M. Marchand-Arvier et al., "In vitro and in vivo evaluation of oral heparin-loaded polymeric nanoparticles in rabbits," Circulation, vol. 105, no. 2, pp. 230-235, 2002.

[17] C. Pazzini, P. D. Marcato, L. B. Prado et al., "Polymeric nanoparticles of enoxaparin as a delivery system: in vivo evaluation in normal rats and in a venous thrombosis rat model," Journal of Nanoscience and Nanotechnology, vol. 15, no. 7, pp. 4837-4843, 2015. 
[18] L. H. Lam, J. E. Silbert, and R. D. Rosenberg, “The separation of active and inactive forms of heparin," Biochemical and Biophysical Research Communications, vol. 69, no. 2, pp. 570-577, 1976.

[19] I. Reyers, L. Mussoni, M. B. Donati, and G. de Gaetano, "Failure of aspirin at different doses to modify experimental thrombosis in rats," Thrombosis Research, vol. 18, no. 5, pp. 669-674, 1980.

[20] I. Carvalho, I. A. Thomazine, F. H. A. Maffei, P. R. Curi, and L. Mattar, "Trombose venosa experimental por ligadura de veia cava no rato: comparação entre diferentes técnicas," in Proceedings of the 7th Annual Meeting of the Federation of Experimental Biology Societies, p. 69, Caxambu, Brazil, 1992.

[21] J. M. Herbert, A. Bernat, and J. P. Maffrand, "Importance of platelets in experimental venous thrombosis in the rat," Blood, vol. 80, no. 9, pp. 2281-2286, 1992.

[22] A. E. Gulyaev, S. E. Gelperina, I. N. Skidan, A. S. Antropov, G. Y. Kivman, and J. Kreuter, "Significant transport of doxorubicin into the brain with polysorbate 80 -coated nanoparticles," Pharmaceutical Research, vol. 16, no. 10, pp. 1564-1569, 1999.

[23] W. Sun, S. Mao, Y. Wang et al., "Bioadhesion and oral absorption of enoxaparin nanocomplexes," International Journal of Pharmaceutics, vol. 386, no. 1-2, pp. 275-281, 2010.

[24] F. T. Meng, G. H. Ma, W. Qiu, and Z. G. Su, "W/O/W double emulsion technique using ethyl acetate as organic solvent: effects of its diffusion rate on the characteristics of microparticles," Journal of Controlled Release, vol. 91, no. 3, pp. 407-416, 2003.

[25] P. R. Klokkevold, D. S. Lew, D. G. Ellis, and C. N. Bertolami, "Effect of chitosan on lingual hemostasis in rabbits," Journal of Oral and Maxillofacial Surgery, vol. 49, no. 8, pp. 858-863, 1991. 

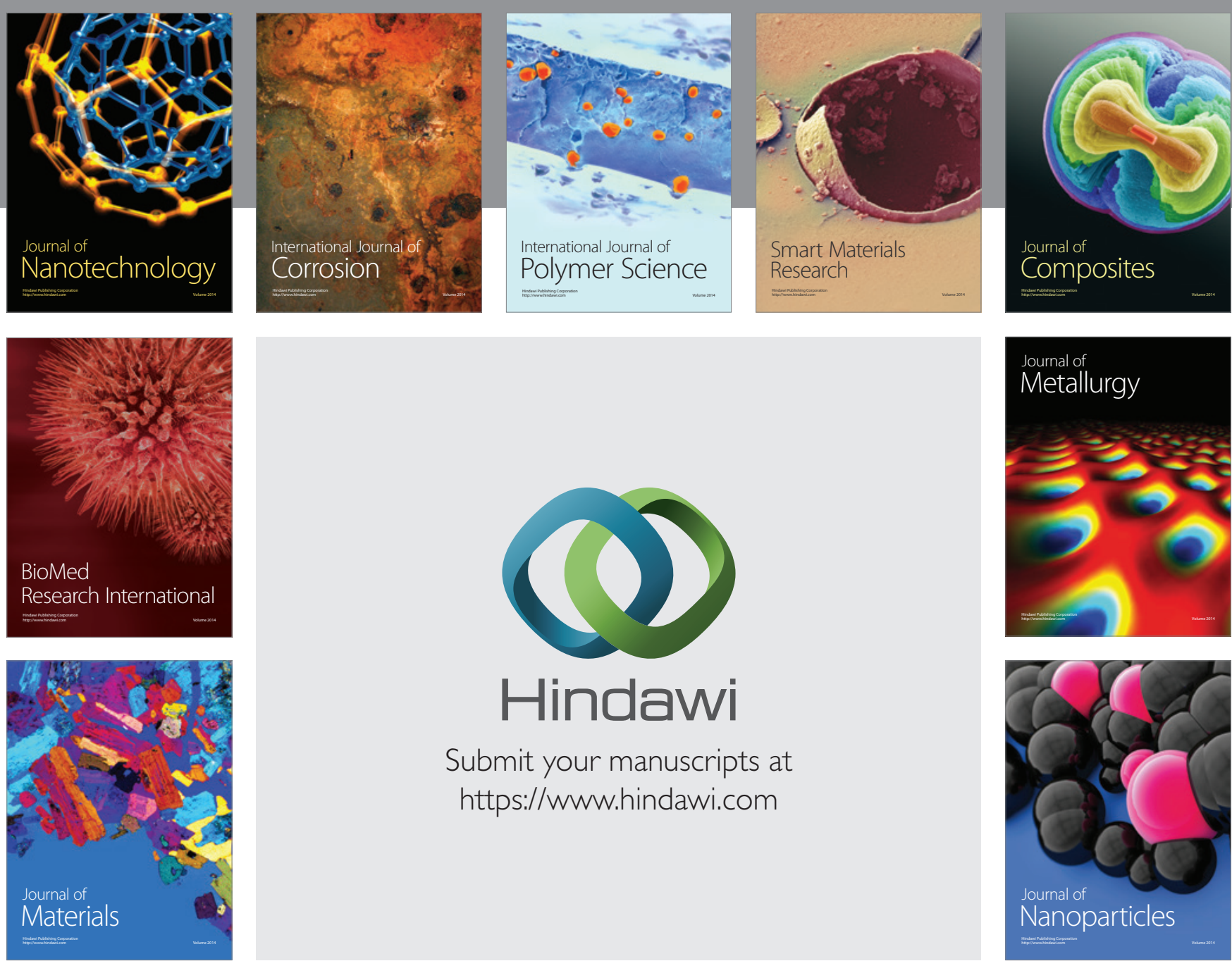

\section{Hindawi}

Submit your manuscripts at

https://www.hindawi.com

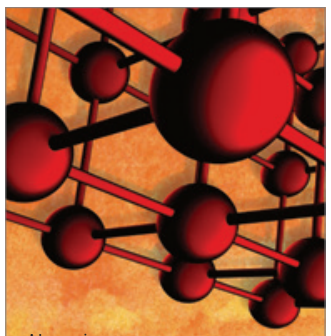

Materials Science and Engineering
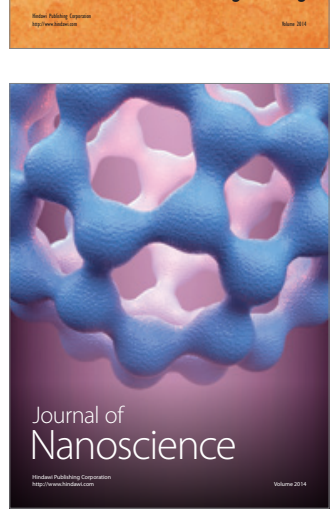
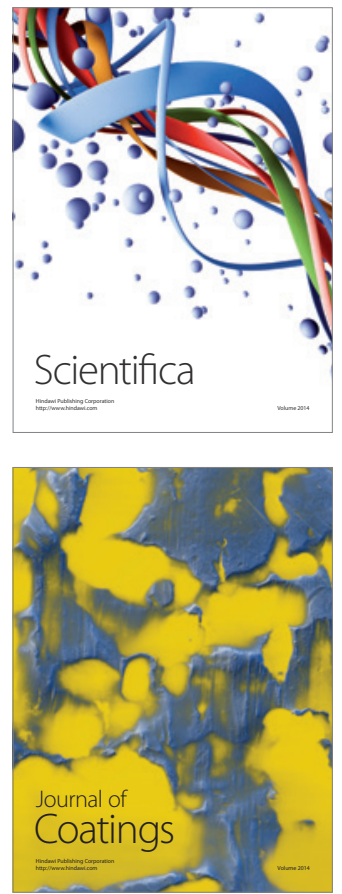
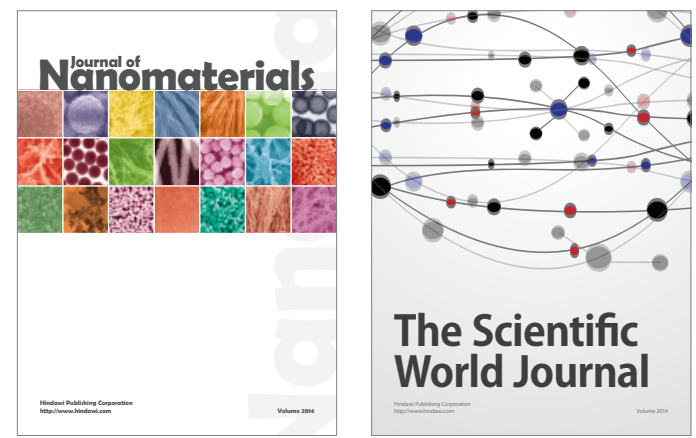

The Scientific World Journal
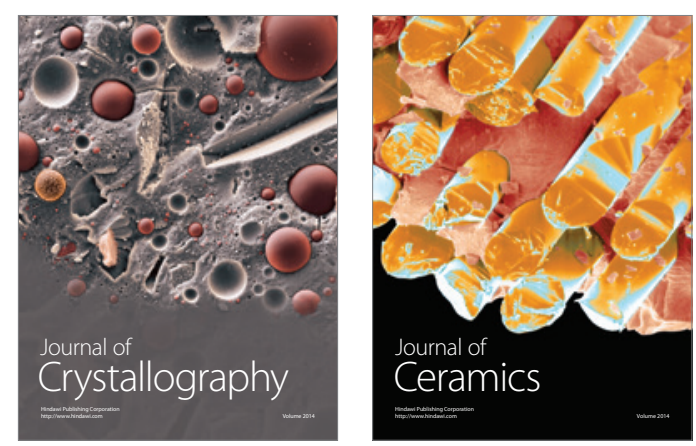
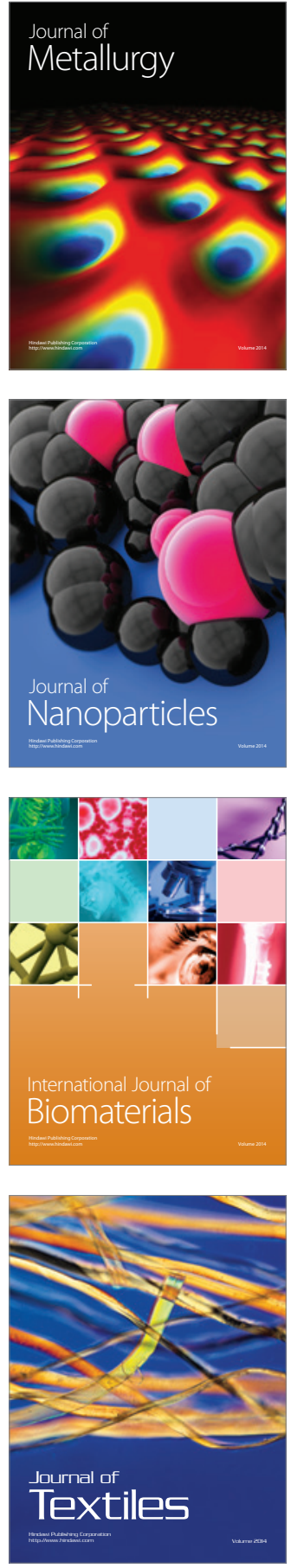